1'Departamento de Análises Clínicas e Toxicológicas, Universidade Federal do Rio Grande do Norte (UFRN), Natal, RN, Brasil

${ }^{2}$ Centro de Educação e Saúde, Universidade Federal de Campina Grande (UFCG), Cuité, PB, Brasil

${ }^{3}$ Departamento de Pediatria, Universidade Federal do Rio Grande do Norte (UFRN), Natal, RN, Brasil ${ }^{4}$ Departamento de Análises Clínicas e Toxicológicas, Universidade de São Paulo (USP), São Paulo, SP, Brasil

Correspondence to: Adriana Augusto de Rezende Av. General Gustavo

Cordeiro de Farias, $s / n$

Faculdade de Farmácia 59012-570 - Natal, RN, Brasil adrirezende@yahoo.com

Received on Aug/9/2017 Accepted on May/9/2018

DOI: $10.20945 / 2359-3997000000057$

\section{The low-density lipoprotein receptor-related protein 5 (LRPS) 4037C >T polymorphism: candidate for susceptibility to type 1 diabetes mellitus}

\begin{abstract}
Karla Simone Costa de Souza', Marcela Abbott Galvão Ururahy', Yonara Monique da Costa Oliveira',2, Melina Bezerra Loureiro', Heglayne Pereira Vital da Silva', Raul Hernandes Bortolin', André Ducati Luchessi', Ricardo Fernando Arrais ${ }^{3}$, Rosário Dominguez Crespo Hirata ${ }^{4}$, Maria das Graças Almeida', Mário Hiroyuki Hirata ${ }^{4}$, Adriana Augusto de Rezende'
\end{abstract}

\begin{abstract}
Objective: The present study has investigated the association between low-density lipoprotein receptor-related protein 5 (LRP5) 4037C>T polymorphism and type 1 diabetes mellitus (T1DM) susceptibility in a Brazilian population. Subjects and methods: A total number of 134T1DM patients and 180 normoglycemic individuals (NG) aged 6-20 years were studied. Glycated hemoglobin and glucose levels were determined. Genotyping of LRP5 4037C >T (rs3736228) was performed. Results: T1DM patients showed poor glycemic control. Genotypes in the codominant (CT: OR $=2.99$ [Cl 95\%: 1.71-5.24], $p<0.001$; TT: OR $=5.34$ [Cl 95\%: 1.05-27.02], $p<0.001$ ), dominant (CT + TT: OR = 3.16 [Cl 95\%: 1.84-5.43], $p<0.001)$ and log-additive (OR $=2.78$ [Cl 95\%: 1.70-4.52], $p<0.001)$ models, and LRP5 4037T allele (OR $=2.88,[\mathrm{Cl} 95 \%$ : 1.78-4.77], $p<0.001)$ were associated with an increased risk of developing T1DM. LRP5 4037CT and CT+TT carriers in T1DM group showed higher concentrations of serum glucose and glycated hemoglobin when compared with CC carriers. Conclusion: The LRP5 4037C > T may represent a candidate for T1DM susceptibility, as well as poor glycemic control. Arch Endocrinol Metab. 2018;62(4):480-4
\end{abstract}

Keywords

Type 1 diabetes mellitus; LRP5; 4037C>T; rs3736228

\section{INTRODUCTION}

$\mathrm{T}$ ype 1 diabetes mellitus (TIDM) is a chronic autoimmune disorder that develops in susceptible individuals by environmental and genetic factors (1-3). Among the genetic factors, besides the HLA region on chromosome $6 \mathrm{q} 21$, which contributes to approximately $40 \%$ of TIDM development, more than 50 non-HLA genes significantly increase the risk of TIDM $(1,2,4)$. Throughout nearly four decades since this discovery, researchers have investigated other genetic loci, in addition to HLA, that could contribute to the risk of $\operatorname{TlDM}(5,6)$.

Among several other mapped loci are those on chromosome llql3.4 $(7,8)$. Within the chromosome region linked to and associated with TIDM, the member of the LDL receptor family, low-density lipoprotein receptor-related protein 5 (LRP5) has been identified $(5,7,8)$. LRP5 is composed of a rapidly expanding number of structurally related proteins which serve a variety of functions in lipid metabolism and signal transduction to participate in cell proliferation (7-9). In addition, LRP5 is a membrane protein found in many tissues and cells, such as pancreatic beta cells, being essential for the insulin hormone production signaling pathway (10).

Fujino and cols. (10) studied the role of LRP5 in pancreatic beta cells through animal model and found that the lack of the gene encoding LRP5 disrupts 
$\beta$-catenin activity by deleting the LRP5, a co-receptor involved in $\beta$-catenin-dependent wingless-ints (Wnt) signaling, greatly impairing beta cell proliferation, as well as glucose-stimulated insulin secretion, which coincides with reduced levels of intracellular ATP and calcium in response to glucose. Moreover, polymorphisms and mutations in the LRP5 are associated with osteoporosis, impaired glucose, increased risk for obesity, type 2 diabetes mellitus, and other metabolic diseases $(7-9,11,12)$.

Studies performed in diabetic families for scan of candidate genes of the TIDM, have indicated that LRP5 missense mutations can trigger a non-coding LRP5, which could be associated with dysfunction in activity beta cells, lack of insulin production, and consequently TIDM development (7-9). However, to the best our knowledge, there are only a few studies associating the LRP5 polymorphism and T1DM susceptibility (9).

Therefore, considering missense polymorphism LRP5 $4037 \mathrm{C}>\mathrm{T}$, characterized by a substitution of alaninevaline amino acids in position 1330 (Alal330Val), this study aimed to investigate the association between LRP5 4037C $>\mathrm{T}$ and TIDM susceptibility in children and adolescents in a Brazilian population.

\section{SUBJECTS AND METHODS}

\section{Study population}

We recruited 134 TIDM patients between 6 and 20 years old from the Hospital Onofre Lopes (HUOL) of the Federal University of Rio Grande do Norte (UFRN), Natal, RN, Brazil from January 2010 to June 2011. TIDM diagnoses were in accordance to the American Diabetes Association (ADA) criteria (3). All patients had a typical history of hyperglycemia and required insulin treatment continuously upon diagnosis. The study was conducted in accordance with the guidelines set by the Ethics in Research Committee of HUOL/UFRN, which complies with the Declaration of Helsinki (protocol number 704 310). One hundred eighty unrelated subjects with no previous diagnosis of TIDM, with fasting serum glucose $\leq 99 \mathrm{mg} / \mathrm{dL}$ (Normoglycemic Group - NG) and the same age range were recruited in local public schools of Natal, RN, Brazil. Informed consent was obtained from all T1DM and NG subjects or their parents. After taking a medical history and performing a physical examination, blood samples were collected for biochemical analysis and DNA extraction.

\section{Laboratory procedures}

Glycemic control was assessed by the determination of glycated hemoglobin in total blood and serum glucose concentration. Both tests were performed using LABTEST kits (Lagoa Santa, Brazil) and LABMAX PLENNO equipment (LABTEST) for glucose and RA 50 spectrophotometer (Bayer Diagnostics, Dublin, Ireland) for glycated hemoglobin.

\section{LRP5 genotyping}

DNA was obtained from peripheral blood mononuclear cell (PBMC), previously isolated by density gradient centrifugation, using Ficoll-Hypaque (Sigma-Aldrich, MO, USA; density $1.077 \mathrm{~g} / \mathrm{mL}$ ). DNA was extracted using the commercial kit Illustra Triple Prep ${ }^{\circledR}$ (GE Healthcare, Little Chalfont, Buckinghamshire, UK), according to the manufacturer's protocol.

DNA integrity was evaluated by electrophoretic separation on a $0.8 \%$ agarose gel in TBE buffer $(\mathrm{pH}$ $=8.0$ ) and stained with GelRed ${ }^{\mathrm{TM}}$ (Uniscience, São Paulo, SP, Brazil). DNA was quantified (A260nm) and had its purity (A260/A280) assessed by ultraviolet spectrophotometry, through the ND-1000 spectrophotometer (NanoDrop Technologies Inc., Wilmington, DE, USA).

LRP5 4037C >T (rs3736228) polymorphism was performed by TaqMan ${ }^{\circledR}$ allelic discrimination on a 7500 Fast Real-Time PCR System (Applied Biosystems, Foster City, CA, USA). LRP5 4037C>T was detected using the Applied Biosystems TaqMan ${ }^{\circledR}$ pre-designed assay C_25752205_10.

\section{Statistical analysis}

Distribution of variables was analyzed by the Kolmogorov-Smirnov test. Variables with normal distributions were subjected to a t-test, and those skew-distributed were analyzed by Mann-Whitney's test. Differences between the sex, Hardy-Weinberg equilibrium, and allele/genotype frequencies were tested by a $\chi^{2}$ analysis test. Those tests were performed with SPSS version 15.0 (SPSS Inc., Chicago, IL, USA).

The logistic regression model was applied to analyze the genotypes according to the genetic models: codominant, dominant, overdominant, recessive, and log-additive (13). These analyses were assessed according to the goodness of fit evaluated by Akaike information criterion (AIC) value and Bayesian information criterion (BIC) $(14,15)$. Odds ratio (OR), confidence interval (CI) 
of $95 \%$, and their corresponding $p$-values were calculated to evaluate the study variables and their influence on TIDM onset. For LRP5 4037C >T polymorphism, $p$-value was computed using the likelihood ratio test. For this analysis, the $\mathrm{R}$ program library SNPassoc version 2.12.2 (R Foundation for Statistical Computing, Vienna, Austria) (16) was used and the reference genotype was homozygous for the wild allele among controls.

Tests with $p$-values $<0.05$ were considered statistically significant.

\section{RESULTS}

Table 1 shows characteristics of NG and TIDM patients. As expected, serum glucose and glycated hemoglobin values were significantly higher in the TIDM group compared to NG individuals $(p<0.001)$. Furthermore, glucose and glycated hemoglobin values in the TIDM group were higher than those recommended by the ADA for good glycemic control in the same age range of the studied individuals (glycated hemoglobin: $\geq 7.5 \%$ ).

The Hardy-Weinberg equilibrium was verified. Ten percent of the samples were re-genotyped at random to assure scoring quality, and all results were consistent.

Allele frequency for $L R P 54037 \mathrm{C}>\mathrm{T}$ is demonstrated in Table 2. A significant increase in the frequency of the LRP5 4037T allele was found in T1DM compared to the NG group $(p<0.001)$.

Considering the genotype distribution of LRP5 $4037 \mathrm{C}>\mathrm{T}$ in the $\mathrm{NG}$ and TIDM groups according to the genetic model, a significant association with TIDM susceptibility was found for codominant $(p<0.001)$,

Table 1. Characteristics of normoglycemic and diabetic patients

\begin{tabular}{lccc}
\hline Variables & NG $\mathbf{~}=\mathbf{1 8 0}$ & T1DM $\mathbf{~ = 1 3 4}$ & $\boldsymbol{p}$-value \\
\hline Biodemographic characteristics & & & 0.939 \\
Sex, Female, \% & 57.8 & 58.2 & 0.876 \\
Age, years & $11.0(9.0-15.0)$ & $12.0(9.0-15.0)$ & - \\
Age at diagnosis, years & - & $6.5 \pm 3.5$ & - \\
Time since diagnosis, years & - & $4.0(2.0-7.0)$ & \\
Glycemic control & & & $\mathbf{0 . 0 0 1}$ \\
Glucose, mg/dL & $79.0(73.3-85.8)$ & $208.0(130.0-316.0)$ & $<\mathbf{0 . 0 0 1}$ \\
Glycated hemoglobin, \% & $5.6(4.8-6.4)$ & $9.6(7.9-12.3)$ & \\
\hline
\end{tabular}

Results are expressed as mean \pm standard deviation or median (interquartile range). n: number of individuals; NG: normoglycemic group; T1DM: type 1 diabetes mellitus group. Significant $p$-values are shown in bold.

Table 2. Allele frequencies and genotype distribution of LRP5 4037CST polymorphism in the studied groups according to the genetic model

\begin{tabular}{|c|c|c|c|c|c|c|}
\hline Polymorphism & & NG n (\%) & T1DM n (\%) & OR (95\% Cl) & $p$-value & AIC \\
\hline \multirow{16}{*}{ LRP5 4037C>T } & Allele & & & & & \\
\hline & C & 331 (91.9) & 214 (79.9) & 1.00 & & \\
\hline & $T$ & $29(8.1)$ & $54(20.1)$ & $2.88(1.78-4.77)$ & $<0.001$ & - \\
\hline & Genetic Model & & & & \multirow{5}{*}{$<0.001$} & \multirow{5}{*}{415.8} \\
\hline & Codominant & & & & & \\
\hline & CC & $153(85.0)$ & 86 (64.2) & 1.00 & & \\
\hline & CT & 25 (13.9) & $42(31.3)$ & $2.99(1.71-5.24)$ & & \\
\hline & $\pi$ & $2(1.1)$ & $6(4.5)$ & $5.34(1.05-27.02)$ & & \\
\hline & Dominant & & & & \multirow{3}{*}{$<0.001$} & \multirow{3}{*}{414.3} \\
\hline & $\mathrm{CC}$ & $153(85.0)$ & $86(64.2)$ & 1.00 & & \\
\hline & $\mathrm{CT}+\mathrm{TT}$ & 27 (15.0) & 48 (35.8) & $3.16(1.84-5.43$ & & \\
\hline & Recessive & & & & & \\
\hline & $\mathrm{CC}+\mathrm{CT}$ & 178 (98.9) & $128(95.5)$ & 1.00 & & \\
\hline & $\Pi T$ & $2(1.1)$ & $6(4.5)$ & $4.17(0.83-21.00)$ & 0.060 & 429.0 \\
\hline & Log-additive & & & & & \\
\hline & & $180(57.3)$ & $134(42.7)$ & $2.78(1.70-4.52)$ & $<0.001$ & 414.1 \\
\hline
\end{tabular}

NG: normoglycemic group; T1DM: type 1 diabetes mellitus group; $n$ : number of individuals; OR: odds ratio; Cl: confidence interval; AIC: Akaike's Information Criterion. Significant $p$-values are shown in bold. 
dominant $(p<0.001)$ and log-additive $(p<0.001)$ models (Table 2).

In this study, also was investigate the influence of LRP5 4037C $>\mathrm{T}$ in the parameters of glycemic control. Significantly increased values of serum glucose and glycated hemoglobin were observed in the LRP5 4037CT [glucose mean: $230.2 \mathrm{mg} / \mathrm{dL}(p<0.001)$; glycated hemoglobin mean: 10.3\% $(p=0.017)$ ] and CT+ TT carriers [glucose mean: $227.8 \mathrm{mg} / \mathrm{dL}$ $(p<0.001)$; glycated hemoglobin mean: 10.5\%, $(p=0.002)]$ when compared to the CC genotype carriers [glucose mean: $98.9 \mathrm{mg} / \mathrm{dL}$; glycated hemoglobin mean: $7.3 \%]$.

\section{DISCUSSION}

TIDM is characterized by autoimmune destruction of pancreatic beta cells with multiple genes involved, and among them is LRP5 (3,7-9). The relationship of this gene with TIDM was found in 1998 by Nakagawa and cols., that described only 10 polymorphisms of LRP5 associated with susceptibility to TIDM, after to perform a genomic scanning in 707 diabetic families from the UK, USA, Italy, and Norway. However, data in the literature on this association remains scarce and for our knowledge the present study is the first to find the association between LRP5 4037C>T, T1DM susceptibility, and poor glycemic control.

On the other hand, the LRP $54037 \mathrm{C}>\mathrm{T}$ is recognized as one of the key polymorphism in associated with increase the risk of bone fracture and osteoporosis, as well as in the cardiovascular diseases (12,17-20). In addition, it has been reported that the LRP5 4037C > T is associated with susceptibility to type 2 diabetes mellitus and osteoporosis in postmenopausal women. In this diseases, the genotype $\mathrm{CT} / \mathrm{TT}$ increases the risk of the developing them $(12,21)$.

LRP5 is a member of the LDL receptor superfamily, which was originally cloned on the basis of its genetic association with TIDM in humans, and is found in the $5^{\prime}$ region of the chromosome llql3 (9). The binding of Wnt with LRP5-frizzled receptor triggers LRP5 signaling. This link leads to inactivation of the "complex of destruction" of $\beta$-catenin, formed by proteins of adenomatous polyposis coli (APC), axis inhibition protein (Axin), and glycogen synthase

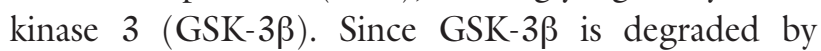
the dishevelled protein, it inhibits the proteasomal degradation of $\beta$-catenin, which accumulates in the cytoplasm and then translocates to the nucleus where it associates with transcription factors such as transcription factor 7, which will control the transcription of target genes for beta cell proliferation $(14,22,23)$. Though there were no mechanistic explanations as to how Wnt signaling was involved in the increased proliferation of beta cell mass and insulin production, studies suggest that LRP5 is essential for these cells $(10,14,22)$.

Several studies have speculated that hyperglycemia may be conferred by changes in LRP5 expression levels or tissue expression specificity, since LRP5 signaling contributes to the glucose-induced insulin secretion in the islets of Langerhans, however this mechanism is unclear $(10,22)$. These studies raised the possibility that polymorphisms would favor the negative regulation of transcription or specificity of this gene on pancreatic beta-cells, hampering glucose-induced insulin secretion (10,22). A study using an animal model indicated that mice deficient of LRP5 had low levels of insulin-signaling proteins for mRNA expression, such as the insulin receptor. Impaired glucose tolerance, as well as high glucose concentrations were found, demonstrating the importance of LRP5 in this signaling (10). In addition, studies have associated LRP5 missense mutations with the TIDM onset, once these mutations could change the pancreatic beta cell signaling, impairing insulin secretion (7-9,22).

Our results show an association between LRP5 $4037 \mathrm{C}>\mathrm{T}$ polymorphism and T1DM susceptibility. Furthermore, individuals who were carriers of CT and CT + TT genotypes had higher concentrations of serum glucose and glycated hemoglobin than individuals with the wild-type homozygous genotype. Thus, according Iniesta and cols. (13), the significant association found in codominant, dominant, and log-additive models demonstrate that each additional copy of the variant allele increases the TIDM susceptibility. Additionally, only one copy of the mutated $\mathrm{T}$ allele is sufficient to contribute to poor glycemic control.

In conclusion, our results demonstrated an association of the $\mathrm{T}$ allele for the LRP5 $4037 \mathrm{C}>\mathrm{T}$ polymorphism with TIDM susceptibility in a Brazilian population, moreover the possible relation in the progressive failure of glycemic control.

Acknowledgments: we are grateful for the technical support provided by students from Multidisciplinary laboratory (LABMULT)/Federal University of Rio Grande do Norte (UFRN)/ $\mathrm{RN}$, Brazil. We also thank all the physicians, nurses, and hospital staff at Hospital Onofre Lopes of the UFRN who were involved in the study. The authors thank all children and adolescents with 
TIDM and their parents who gave their consent to participate in the study.

Funding: this study was supported by a grant from the National Counsel of Technological and Scientific Development (CNPq) (620099/2008-9).

Disclosure: no potential conflict of interest relevant to this article was reported.

\section{REFERENCES}

1. Silva HPV, Ururahy MAG, Souza KSC, Loureiro MB, Oliveira YMC, Oliveira GHM, et al. The association between the HLA-G 14-bp insertion/deletion polymorphism and type 1 diabetes. Genes Immun. Genes Immun. 2016;17(1):13-8.

2. van Belle TL, Coppieters KT, von Herrath MG. Type 1 diabetes: etiology, immunology, and therapeutic strategies. Physiol Rev. 2011;91(1):79-118.

3. American Diabetes Association. Standards of Medical Care in Diabetes - 2017. Diabetes Care. 2017;40(Suppl. 1):S1-35.

4. ZemunikT, Boraska V. Genetics ofType 1 Diabetes. World J Diabetes. Split, Croatia: InTech; 2013;4(4):114-23.

5. Ram R, Mehta M, Nguyen QT, Larma I, Boehm BO, Pociot F, et al. Systematic Evaluation of Genes and Genetic Variants Associated with Type 1 Diabetes Susceptibility. J Immunol. 2016 Apr 1;196(7):3043-53.

6. Noble JA, Erlich HA. Genetics of type 1 diabetes. Cold Spring Harb Perspect Med. 2012;2(1):a007732.

7. Twells RC, Mein CA, Payne F, Veijola R, Gilbey M, Bright M, et al. Linkage and association mapping of the LRP5 locus on chromosome 11q13 in type 1 diabetes. Hum Genet. 2003;113(2):99-105.

8. Twells RCJ, Mein CA, Phillips MS, Hess JF, Veijola R, Gilbey M, et al. Haplotype structure, LD blocks, and uneven recombination within the LRP5 gene. Genome Res. 2003;13(5):845-55.

9. Nakagawa Y, Kawaguchi Y, Twells RC, Muxworthy C, Hunter KM, Wilson $A$, et al. Fine mapping of the diabetes-susceptibility locus, IDDM4, on chromosome 11q13. Am J Hum Genet. 1998;63(2): 547-56.

10. Fujino T, Asaba H, Kang M, Ikeda Y, Sone H, Takada S, et al. Lowdensity lipoprotein receptor-related protein 5 (LRP5) is essential for normal cholesterol metabolism and glucose-induced insulin secretion. Proc Natl Acad Sci U S A. 2003;100(1):229-34.
11. Saarinen A, Saukkonen T, KiveläT, Lahtinen U, Laine C, Somer M, et al. Low density lipoprotein receptor-related protein 5 (LRP5) mutations and osteoporosis, impaired glucose metabolism and hypercholesterolaemia. Clin Endocrinol (Oxf). 2010;72(4):481-8.

12. Gay A, Towler DA. Wnt signaling in cardiovascular disease. Curr Opin Lipidol. 2017;28(5):387-96.

13. Iniesta R, Guinó $E$, Moreno V. Análisis estadístico de polimorfismos genéticos en estudios epidemiológicos. Gac Sanit. 2005;19(4):333-41.

14. Minelli C, Thompson JR, Abrams KR, Thakkinstian A, Attia J. The choice of a genetic model in the meta-analysis of molecular association studies. Int J Epidemiol. 2005;34(6):1319-28.

15. Zintzaras E, Lau J. Synthesis of genetic association studies for pertinent gene-disease associations requires appropriate methodological and statistical approaches. J Clin Epidemiol. 2008;61(7):634-45.

16. González JR, Armengol L, Solé X, Guinó E, Mercader JM, Estivill $X$, et al. SNPassoc: an $R$ package to perform whole genome association studies. Bioinformatics. 2007;23(5):644-5.

17. Canto-Cetina T, Polanco Reyes L, González Herrera L, Rojano-Mejía D, Coral-Vázquez RM, Coronel A, et al. Polymorphism of LRP5, but not of TNFRSF11B, is associated with a decrease in bone mineral density in postmenopausal Maya-Mestizo women. Am J Hum Biol. 2013;25(6):713-8.

18. Kitjaroentham A, Hananantachai $H$, Phonrat B, Preutthipan S, Tungtrongchitr R. Low density lipoprotein receptor-related protein 5 gene polymorphisms and osteoporosis in Thai menopausal women. J Negat Results Biomed. 2016;15(1):16.

19. Liu K, Tan L-J, Wang P, Chen X-D, Zhu L-H, Zeng Q, et al. Functional relevance for associations between osteoporosis and genetic variants. PLoS One. 2017;12(4):e0174808.

20. Xu G-Y, Qiu Y, Mao H-J. Common Polymorphism in the LRP5 Gene May Increase the Risk of Bone Fracture and Osteoporosis. Biomed Res Int. 2014;2014:290531.

21. Xuan M, Wang Y, Wang W, Yang J, Li Y, Zhang X. Association of LRP5 gene polymorphism with type 2 diabetes mellitus and osteoporosis in postmenopausal women. Int J Clin Exp Med. 2014;7(1):247-54.

22. Figueroa DJ, Hess JF, Ky B, Brown SD, Sandig V, Hermanowski-Vosatka $A$, et al. Expression of the type I diabetes-associated gene LRP5 in macrophages, vitamin A system cells, and the Islets of Langerhans suggests multiple potential roles in diabetes. $J$ Histochem Cytochem. 2000;48(10):1357-68.

23. Wang $Y$, Chang H, Rattner A, Nathans J. Frizzled Receptors in Development and Disease. CurrTop Dev Biol. 2016;117:113-39. 